Results The demographic information for 132 subjects showed that subjects with $\mathrm{CAD}$ tended to have more unfavourable lipoprotein variables. Genotype distributions at both sites were different between the CAD and control groups. The apo E gene alleles were associated with the plasma levels of lipids and lipoproteins (all $\mathrm{p}<0.05$ ); The control group had higher apo $\mathrm{E} \varepsilon_{2}$ " frequencies than the CAD group $(p<0.001)$ and " $\varepsilon_{2}$ " was significantly correlated with occurrence of $\mathrm{CAD}(\mathrm{p}<0.001)$.

Conclusion The results suggest that the apo E gene polymorphism do have influence circulating levels of lipids and lipoproteins and that individuals with apo $\mathrm{E}$ " $\varepsilon_{2}$ " are likely to have a reduced risk of developing $\mathrm{CAD}$ in northern Chinese.

\section{e0302 EFFECTS OF XUEZHIKANG ON BLOOD LIPIDS AND THE LEVELS OF PLASMA ENDOTHELINS, THROMBOXANE B2, 6-KETO-PGF-1A IN PATIENTS WITH PRIMARY HYPERLIPIDAEMIA}

doi:10.1136/hrt.2010.208967.302

Wei Liao, Yong-ling Liao. Department of Cardiology, The Affiliated Hospital of Gannan Medical College

Objective To observe the effects of Xuezhikang on blood lipids and the levels of plasma endothelins, thromboxane $\mathrm{B}_{2}$, 6-keto-PGFla in patients with primary hyperlipidaemia.

Methods 120 patients with primary hyperlipidaemia were enrolled in this study, 82 males and 38 females, age 36-74 years old, average ages (55 \pm 9$)$ years old. 12 weeks after taking Xuezhikang, the clinical effect and the effect on the level of plasma endothelins, thromboxane $\mathrm{B}_{2}$, 6-Keto-PGFla were compared before and after the treatment, and the relation between blood lipids and ratio of plasma endothelins, thromboxane $\mathrm{B}_{2}$ to 6- Keto-PGFla were analysed.

Results 12 weeks after treatment, the level of TG, cholesterol (TC), low density lipoprotein cholesterol (LDL-C) and $\mathrm{apoB}_{100}$ decreased sharply $(p<0.05-0.001)$; the level of serum high density lipoprotein cholesterol (HDL-C) elevated $(p<0.05)$; ratio of plasma endothelins decreased sharply $(p<0.001)$; rate value of thromboxane $B_{2}$ to 6-Keto-PGFla before treatment was higher than health people but lower after treatment $(p<0.01)$. There were positive correlations between the decreased TC. TG, LDL-C and decreased ET-1, the ratio $\mathrm{TXB}_{2} / 6$-keto-PGFla $(\mathrm{r}=0.832-0.963, \mathrm{p}<0.01-0.001)$. The same positive correlation was found between the decreased ET-1 and the ratio of $\mathrm{TXB}_{2} / 6$-keto-PGF1a $(\mathrm{r}=0.987, \mathrm{p}<0.001)$.

Conclusions Plasma endothelins level and ratio of thromboxane $\mathrm{B}_{2}$ to 6-Keto- PGFla increased in the patients with primary hyperlipidaemia. Xuezhikang not only effectively adjusted blood lipids level but also reduced plasma endothelins level and ratio of thromboxane $\mathrm{B}_{2}$ to 6-Keto- PGFla.

\section{e0303 SIGNIFICANCE OF OXIDISED LOW-DENSITY LIPOPROTEIN IN CORONARY ATHEROSCLEROTIC HEART DISEASE}

doi:10.1136/hrt.2010.208967.303

Li Chang, Meng xiaoping. Jilin University No 2 Hospital

Objective In this study, control group are people with normal coronary arteries. This study is to discuss the relationship between LDL, OX-LDL and control group, SAP group, UAP group, AMI group and contraction of LDL and OX-LDL.

Methods Experimental group are $300 \mathrm{CHD}$ patients without taking lipid-lowering drugs in one month who admitted in the department of cardiolysis from August 2008 to August 2009. They are divided into SAP group (100 cases), UAP group (100 cases), AMI (100 cases). The control group are 100 cases of patients who are randomly selected and confirmed without CHD by coronary angiography in the same period in our department. Information includes gender, age, smoking, drinking, hypertension, diabetes, levels of LDL and OX-LDL. We analysis patients' basic information, the level and correlation of LDL and OX-LDL in control group, SAP group, UAP group and AMI group.

Results 1 . There are no significant difference between control group and CHD groups in basic information $(p>0.05)$. 2. Concentration of $\mathrm{LDL}$ in CHD group is significant difference compared with control group $(p<0.01)$. Concentration of LDL in UAP group is no significant difference compared with SAP group $(p>0.05)$. Concentration of LDL in AMI group is significant difference compared with SAP group $(p<0.01)$. Concentration of LDL in AMI group is no significant difference compared with UAP group $(p>0.05)$. 3. Concentration of OX-LDL in CHD group is significant difference compared with control group $(p<0.01)$. Concentration of OX-LDL in UAP group is significant difference compared with SAP group $(p<0.05)$. Concentration of OX-LDL in AMI group is significant difference compared with SAP group $(p<0.01)$. Concentration of OX-LDL in AMI group is significant difference compared with UAP group $(p<0.01)$. 4. There is no correlation between concentration of LDL and OX-LDL in all groups ( $\mathrm{p}>0.05)$.

Conclusions 1 . We confirm that $\mathrm{LDL}$ and $\mathrm{OX}-\mathrm{LDL}$ are risk factors for $\mathrm{CHD}$. There are no significant differences about the concentration of LDL in CHD groups, however, there is significant differences about the concentration of OX-LDL in CHD groups. The level of OX-LDL is in escalating trend. 2. There is no correlation between concentration of LDL and OX-LDL in all groups $(p>0.05)$ and OX$\mathrm{LDL}$ play a more important role in the process of $\mathrm{CHD}$. Compared with LDL, mensurating OX-LDL is more meaningful in the treatment and prevention of $\mathrm{CHD}$

\section{e0304 LONG-TERM CORONARY HEART DISEASE RISK ASSOCIATED WITH VERY-LOW-DENSITY LIPOPROTEIN CHOLESTEROL IN CHINESE: THE RESULTS OF A 15-YEAR CHINESE MULTI- PROVINCIAL COHORT STUDY (CMCS)}

doi:10.1136/hrt.2010.208967.304

Jie Ren, Scott Grundy, Jing Liu, Wei Wang, Miao Wang, Jiayi Sun, Jun Liu, Yan Li, Zhaosu Wu, Dong Zhao. Capital Medical University, Affiliated Beijing Anzhen Hospital, Beijing Institute of Heart, Lung \& Blood Vessel Diseases

Aims Few studies have examined very-low-density lipoprotein (VLDL) cholesterol as an independent risk factor of coronary heart disease (CHD) or its combined effects with coexisting cardiac risk factors. The current study examined the association between VLDL cholesterol and the risk of future CHD events.

Methods and results This study reports the association of VLDL cholesterol level and long-term CHD risk, as well as the combined effects of VLDL cholesterol and LDL cholesterol with other cardiovascular disease (CVD) risk factors. The cohort comprises 30,378 participants aged 35-64 years from 11 Chinese provinces. All participants were followed up annually until 2007 . We found $20 \%$ of the sample population had elevated VLDL cholesterol $\geq 30 \mathrm{mg} / \mathrm{dl}$. Elevated VLDL cholesterol levels were found to increase CHD risk by $2.19-3.36$ fold in people with LDL cholesterol within the normal range and presenting no other major risk factors. This effect was exacerbated in those with elevated LDL cholesterol levels, and further increased CHD risk in those displaying three or more risk factors. The population-attributable risk proportion (PAR\%) of CHD associated with VLDL cholesterol was calculated to be $17.3 \%$, higher than that associated with LDL cholesterol alone.

Conclusions In a large Chinese cohort, elevated VLDL cholesterol was found to be significantly associated with elevated CHD risk, similar to that observed with LDL cholesterol. CHD risk was further 